\title{
Factors affecting the maintenance dose of warfarin
}

\author{
A H James, R P Britt, C L Raskino, S G Thompson
}

\begin{abstract}
Aim: To identify the possible factors determining the dose of warfarin prescribed in patients receiving anticoagulant treatment.

Methods: The computerised records of 2305 patients maintained on the drug in seven hospitals were amalgamated and classified into one of seven diagnostic groups. The associations with the dose of warfarin prescribed were investigated by univariate and multiple regression analysis. Differences between hospitals were studied with regard to the coagulometric method and the thromboplastin preparation used.
\end{abstract}

Results: The geometric mean dose of warfarin was $4.57 \mathrm{mg}$ and $5 \%$ of patients were prescribed $10 \mathrm{mg}$ or greater. There was a noticeable decrease in dose with increasing age, which averaged about $6 \mathrm{mg}$ for patients aged 30 but $3.5 \mathrm{mg}$ for those aged 80 . Men required slightly more warfarin than women. Patients with heart disease or atrial fibrillation required lower doses of warfarin, while higher doses were required by patients with deep vein thrombosis. Significant differences in mean warfarin dose among the seven hospitals were evident. These differences could not be explained entirely by the use of different coagulometric methods or thromboplastins.

Conclusion: Clinicians should be aware that older patients need reduced doses of warfarin. The considerable differences in

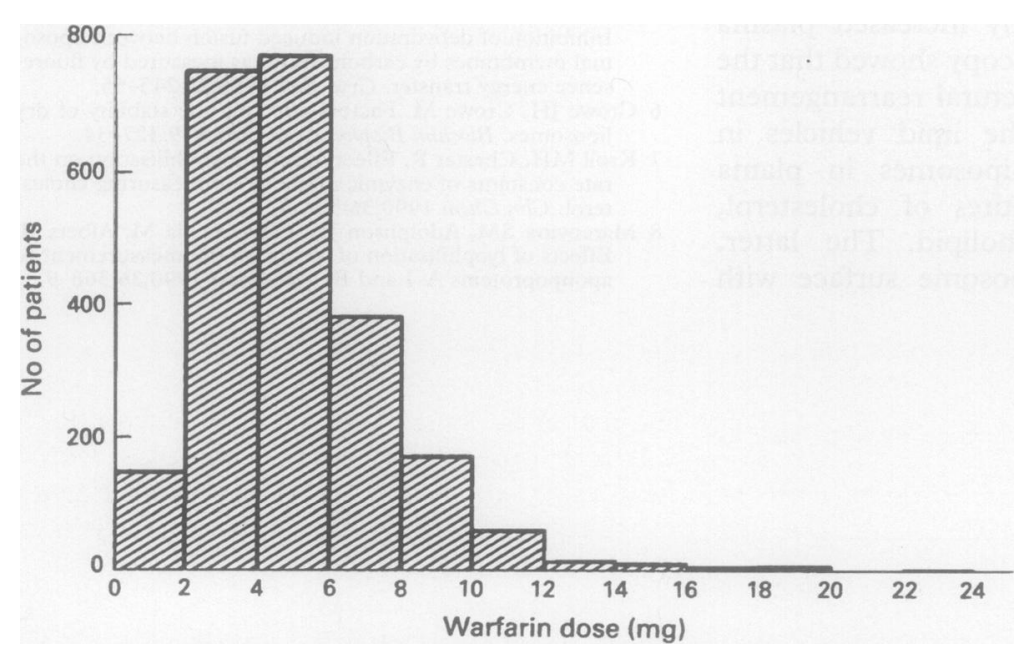

Figure 1 Distribution of warfarin doses prescribed to 2305 patients at seven hospitals. doses of warfarin among hospitals indicates that further efforts to improve uniformity are required.

AC is a software system for personal computers which assists in the outpatient maintenance of warfarin treatment. It is a development of the system originally described by Wilson and James ${ }^{1}$ and keeps records whose primary purpose is to furnish details necessary for the continued management of patients. A by-product, however, is its potential for the study of statistics relating to treatment. As the records produced by the system have a uniform structure, data from several centres can be amalgamated. Of the hospitals using AC, seven kindly allowed us to study their data files, providing records on 2305 patients receiving warfarin.

\section{Methods}

The hospitals contributing data to this study were district general hospitals, six in southern England and one in an offshore island. They had been using the system for periods varying between 10 weeks and seven years. Records of the most recent visit for each patient were, after removal of identifying data, subjected to transformation of date of birth into age and title into sex (with assistance from the first name in a few cases). The diagnostic labels recorded by the hospitals were not identical, but permitted classification of most cases into one of seven groups. There was some loss of specificity in this classification: for example, recurrent embolism was not distinguished from embolism. The target International Normalised Ratio (INR) allocated to each patient, required by the AC system to determine dose changes, was also abstracted from the records.

The associations with the dose of warfarin prescribed were investigated first for characteristics considered singly (univariate analyses) and subsequently in a multiple regression analysis which considered all the factors simultaneously. The distribution of the doses for the 2305 patients is shown in fig 1 . It is positively skewed and so all analyses have been carried out using a logarithmic transformation. The mean values of warfarin dose presented are therefore geometric means.

We investigated how coagulometric method and thromboplastin preparation might relate to systematic differences in average warfarin doses among hospitals. Formally, this was achieved by testing the extent to which varia- 


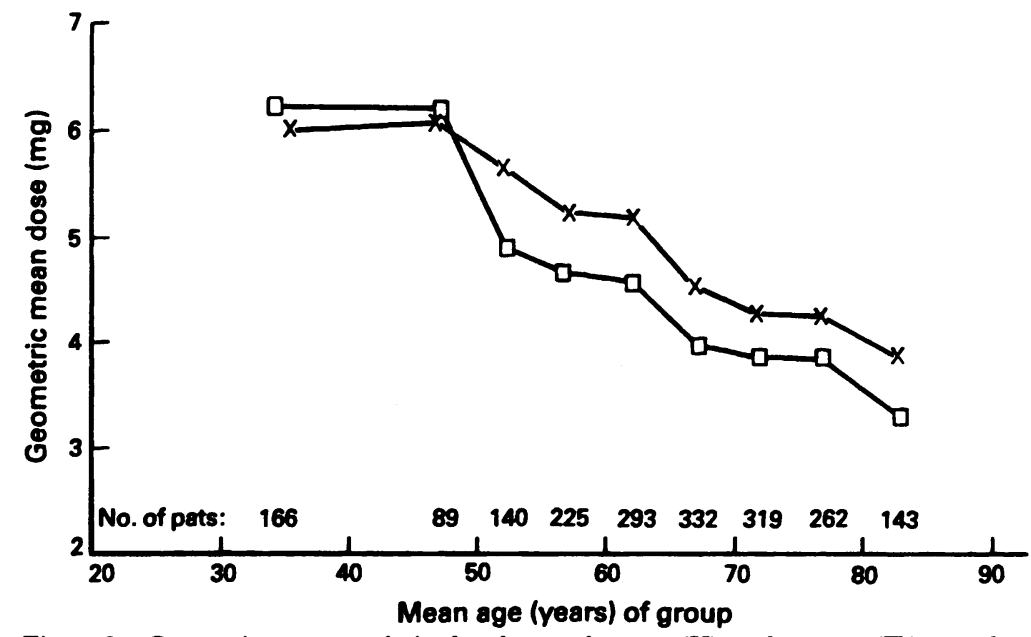

Figure 2 Geometric mean warfarin dose by age for men $(X)$ and women $(\square)$, together with numbers of patients by age group ( $<45$ years, five-year age groups $45-80$, and $\geqslant 80$ years).
Table 2 Geometric mean warfarin dose ( $\mathrm{mg}$ ) by target INR

\begin{tabular}{lll}
\hline Target INR & $N=$ & Mean \\
\hline$<2 \cdot 7$ & 786 & $4 \cdot 34$ \\
$2 \cdot 7-2 \cdot 8$ & 545 & $4 \cdot 35$ \\
$2 \cdot 9-3 \cdot 2$ & 536 & $4 \cdot 57$ \\
$>3 \cdot 2$ & 438 & $5 \cdot 35$ \\
\hline
\end{tabular}

able in terms of different ages or target INRs of men and women (table 1).

Target INR was positively related to warfarin dose, as expected (table 2). The average increase in dose was $11.5 \%$ (95\% confidence interval $8.5 \%-14.5 \%$ ) per 0.5 INR unit $(p<0.0001)$. This relation was maintained in a multiple regression analysis (table 1 ).

The average doses differed slightly between the diagnostic groups (table 3). The small number of patients receiving prophylactic treatment does not reflect practice because such treatment is mainly given to inpatients for whom the AC system is not designed. The group with heart disease or atrial fibrillation had a low average dose, probably due to concomitant drug treatment. The higher dose in the group with prosthetic valve disease was associated with selection of higher target INRs.

The high figure for the group with deep vein thrombosis (DVT) merits particular comment. In view of the large effect of age on dose, a possible reason why patients with DVT need more warfarin could have been an excess of young patients. However, separation of the patients into five year groups showed that for all groups with substantial numbers the DVT patients required more warfarin than patients with other diagnoses. Thus the average dose in DVT patients, after adjusting for age and other variables in a multiple regression (table 1), remained $17 \%$ higher than in other patients.

The average warfarin doses prescribed by the seven hospitals (table 4 ) were significantly different $(p<0.0001)$, the geometric means ranging from $4.17 \mathrm{mg}$ in hospital 5 to $5.19 \mathrm{mg}$ in hospital 3. These differences were maintained in a multiple regression (table 1 ) and so could not be explained by differences between the hospitals in terms of patients' characteristics, such as age, sex, target INR or diagnostic group. The coagulometric method and thromboplastin used by the different hospitals (shown in table 4) were not significantly related to the differences between hospitals in warfarin dose, so that these data do not of themselves establish differences between thromboplastins or coagulometers.

\section{Discussion}

In view of the positively skewed distribution of warfarin doses, results have been presented here as geometric means. Poller and Taberner reported the arithmetic mean dose of warfarin taken by patients in the United Kingdom on long term anticoagulation. ${ }^{2}$ Their figure was obtained from only 120 subjects, being between $4.5 \mathrm{mg}$ and $5.5 \mathrm{mg}$ for six hospitals. with complete data $(n=1755)$

\begin{tabular}{lll}
\hline & Univariate & Multivariate \\
\hline Decrease per decade increase in age & $11.1 \%(\mathrm{p}<0.0001)$ & $9.2 \%(\mathrm{p}<0.0001)$ \\
Increase per 0.5 increase in target INR & $12.4 \%(\mathrm{p}<0.0001)$ & $13.2 \%(\mathrm{p}<0.0001)$ \\
Increase in men compared with women & $12.7 \%(\mathrm{p}<0.0001)$ & $10.0 \%(\mathrm{p}<0.0001)$ \\
Differences between diagnostic groups & $\mathrm{p}<0.0001$ & $\mathrm{p}<0.0001$ \\
Differences between hospitals & $\mathrm{p}<0.0001$ & $\mathrm{p}<0.0005$ \\
\hline
\end{tabular}


Table 3 Geometric mean warfarin dose ( $\mathrm{mg}$ ) by diagnostic group

\begin{tabular}{lrrl}
\hline & $N^{*}$ & Mean & $95 \%$ confidence limits \\
\hline Prosthetic heart valve & 483 & $5 \cdot 22$ & $5 \cdot 03-5 \cdot 43$ \\
Deep vein thrombosis & 398 & $5 \cdot 13$ & $4 \cdot 89-5 \cdot 39$ \\
Pulmonary embolism & 305 & $4 \cdot 64$ & $4 \cdot 39-4 \cdot 90$ \\
Prophylaxis & 21 & $4 \cdot 55$ & $3 \cdot 24-6 \cdot 39$ \\
Arterial disease & 252 & $4 \cdot 54$ & $4 \cdot 27-4 \cdot 83$ \\
Coronary disease & 82 & $4 \cdot 42$ & $3 \cdot 98-4 \cdot 90$ \\
Heart disease with or without atrial fibrillation & 528 & $3 \cdot 91$ & $3 \cdot 75-4 \cdot 07$ \\
Total & 2069 & & \\
\hline
\end{tabular}

Differences between diagnostic groups by one-way ANOVA: $p<0.0001$

*236 patients had missing or unclassifiable diagnostic labels.

Table 4 Geometric mean warfarin dose (mg) by hospital

\begin{tabular}{lcllll}
\hline Hospital & $N=$ & Mean & $\begin{array}{l}\text { 95\% confidence } \\
\text { interval }\end{array}$ & Coagulometer & Thromboplastin \\
\hline 1 & 173 & $4 \cdot 77$ & $4 \cdot 44-5 \cdot 11$ & Coagamate & Manchester \\
2 & 688 & $4 \cdot 71$ & $4 \cdot 55-4 \cdot 88$ & ACL & Manchester \\
3 & 232 & $5 \cdot 19$ & $4 \cdot 84-5 \cdot 56$ & Coagamate & Manchester \\
4 & 327 & $4 \cdot 29$ & $4 \cdot 07-4 \cdot 52$ & Manual & Diagen \\
5 & 357 & $4 \cdot 17$ & $3 \cdot 97-4 \cdot 37$ & ACL & Diagen \\
6 & 123 & $4 \cdot 94$ & $4 \cdot 55-5 \cdot 37$ & ACL & Diagen \\
7 & 405 & $4 \cdot 44$ & $4 \cdot 22-4 \cdot 68$ & KC10 & Diagen \\
Overall & 2305 & $4 \cdot 57$ & $4 \cdot 48-4 \cdot 67$ & & \\
\hline
\end{tabular}

Differences between hospitals by one-way ANOVA: $p<0.0001$

Our arithmetic mean dose was $5 \cdot 13 \mathrm{mg}$, thus falling within their range and suggesting that there has not been a major shift in warfarin dose during the past ten years, despite the change to rabbit thromboplastin in 1986.

Prominent among our findings was the strong negative relation between age and dose. At present, we cannot explain the age dependency of dose, but it should perhaps be taken into account more than it is in judging initial dosage: the BSH Guidelines ${ }^{3}$ say only that the dose schedule should be reduced if the patient is more than $\mathbf{8 0}$ years old. This presumably refers to the initial priming dose, as the ultimate stable dose will be determined by the sensitivity of the patient. Although they refer only to maintenance doses, our data support the implication that older patients should routinely receive a smaller initial dose.

We have shown that there is a significant variation in dose among hospitals. In the light of information from NEQAS coagulation exercises ${ }^{4}$ it was considered that differences might be related to the use of different thrombo- plastin preparations in the quick one-stage method. Over a period of two years (NEQAS Exercises 63-68), all but two of 12 plasma samples tested gave lower results for INR with Manchester reagent than with other reagents (excluding Thromborel). This is clearly apparent in the histograms in the reports. The accentuation of the discrepancy with higher INRs is also seen in the study of Roper et al. ${ }^{5}$ Eight patients receiving warfarin whose INR tested with other reagents was over $5 \cdot 0$ showed values less than 4.0 when tested with two batches of Manchester thromboplastin. Hospitals using Manchester reagent would therefore be expected to give greater doses of warfarin.

A further possible cause of differences among hospitals is the use of coagulometers, some of which have been shown to give lower INRs than others. ${ }^{6}$ However, we did not analyse data from a sufficient number of hospitals to attribute differences either to thromboplastins or coagulometers. What differences there are, however, conform with the above studies: an investigation involving more hospitals would be valuable.

Of interest is the finding of a higher warfarin requirement for patients with deep vein thrombosis compared with those with other diagnoses. Greater body weight may be a factor, but we have no data to check this point. The higher dose may indicate the existence of a hypercoagulable state in some patients, and it would be of interest to examine the requirement of patients with known thrombophilia.

We are much indebted to the consultants and their staff of the hospitals whose records we were able to study.

1 Wilson R, James AH. Computer assisted management of warfarin treatment. Br Med $₹$ 1984;289:422-4.

2 Poller L, Taberner DA. Dosage and control of oral anticoagulants: an international collaborative survey. $\mathrm{Br} \mathrm{F}$ Haematol 1982,51:479-85.

3 British Society for Haematology. British Committee for Standards in Haematology. Haemastasis and Thrombosis Task Force. Guidelines on oral anticoagulation: second edition. $\mathcal{f}$ Clin Pathol 1990;43:177-83.

4 UK National External Quality Assessment Scheme in Blood Coagulation. (1989-90) Reports on Surveys 63-68.

5 Roper JL, Morrell H, Dennis SN, George AJ. Discrepancy between capillary and venous INR values. $\mathrm{Br} \mathcal{F}$ Haematol 1990(Suppl 1);76:18.

6 Thomson JM, Taberner DA, Poller L. Automation and prothrombin time: a United Kingdom field study of two prothrombin time: a United Kingdom field study of two 679-84. 